\title{
Neuroanatomical Background to Understanding the Brain of the Young Psychopath
}

\author{
James H. Fallon*
}

The objective of this paper is to describe the basis of violent psychopathology from a neuroanatomical point of view. Although there is no universally accepted neurological cause of psychopathology, there are some basic patterns of brain malfunction that are present in a majority of cases. Most notably is the compromised functioning of the orbital prefrontal cortex and associated neural circuitry. Beyond this fundamental finding are considerations of how many types of psychopaths there are, how they are characterized, and what may be the potential contributing causes. These questions are approached from multiple levels of neuroanatomical analysis, including genetic, molecular, neurotransmitter, neuronal, cell cluster, and brain area levels, to entire circuit systems. These levels are then considered in light of prenatal, perinatal, and postnatal development and the timing of neuronal system damage in the life of the psychopathic individual. The mechanistic neuroscience approaches are discussed in terms of the relative contributions of nature (genetic/epigenetic/brain damage) and nurture (environmental).

The following conclusions are offered. First, violent psychopathology in youth is associated with structural and functional damage to the orbital cortex and strongly interconnected ventral prefrontal system structures such as the ventromedial prefrontal cortex, ventral anterior cingulate, amygdala, and associated basal ganglia and cortico-subcortical loop circuits. Second, the timing of the injury or malfunctioning of this circuit determines the type of psychopathology manifested later in development, with a) prenatal (e.g., faulty neuronal migration, in utero endocrine toxicity) and perinatal damage occurring prior to two to three years of life associated with a psychopathology where the eventual child/adolescent/adult carries out inappropriately violent acts without knowing what they are doing is wrong, b) damage occurring after the second or third year of life up to about puberty associated with a psychopathology where the eventual child/adolescent/adult carries out violent acts and knows what they are doing is wrong, but they cannot control the impulse to act, and c) damage occurring later in life where severe antisocial acts are carried out with full knowledge of the morality and consequences involved, but with a highly variable ability to inhibit the impulses at some point in time and circumstance. Third, there

- Professor of Anatomy and Neurobiology, College of Medicine, University of California, Irvine, B.A., St. Michael's College, 1969, M.S. Psychology, Rensselaer Polytechnic Institute, 1972, Ph.D. Anatomy, University of Illinois, 1975, Postdoctoral, Neurosciences, University of California, San Diego, 1978. 
are limited treatments for some of the comorbid conditions and associated clinical findings (e.g., impulsivity, aggression, delinquency, cardiovascular anomalies, stress response), but the core violent psychopathic condition appears to still be impenetrable to intervention or prevention. Lastly, the new transdisciplinary field of imaging genetics offers a novel approach to predicting, characterizing, and treating affective, cognitive and behavioral disorders, and may offer a powerful new tool for understanding psychopathology and how it is viewed and adjudicated in the legal system.

\section{INTRODUCTION}

This paper is intended to provide a basic neuroanatomical framework upon which to interpret the range of normal and psychopathic phenotypes that may be encountered during a criminal trial where culpability, especially of an adolescent or young adult defendant, may be at issue. In considering this question from a scientific perspective, there are nevertheless several caveats that must be made about the material that will follow. I have never testified in any criminal trial as either an expert witness or a clinician of any kind; rather I am a research neuroanatomist. Thus, my opinions may seem either extremely obvious or completely irrelevant to the legal expert. Moreover, no one yet really knows what forms the basis for the myriad of psychopathic behaviors, especially those where the degree of free will, intention, and self-control by the accused cannot be fully known. Thus, almost any politically, religiously, or ethically motivated argument could be made and supported in the reference material. ${ }^{1}$

Even within my own field of "wet neuroscience," opinions are staggeringly different. For example, social neuroscientists, cognitive neuroscientists, behavioral psychologists, and psychotherapists may, as a group, hold that behavior, including psychopathic behavior, falls under environmental (nurture) controls. On the other hand, molecular, genetic, pharmacological, and anatomically-minded neuroscientists may, as a group, hold that behavior falls under genetic and epigenetic (nature) controls. After a half a century of dominance by the environmental theorists, the past two decades have witnessed a shift toward more genetically weighted theories, although it has become politically correct in the last decade to say that both genetics and the environment interact to form the basis for behavior. This seemingly simple proposition, however, may be of limited use when seeking to determine the culpability of a violent juvenile offender.

It also is important to note that no scientist or non-scientist really knows how the brain works, or how thought, emotion, and behaviors emerge from the interaction of brain connections and the other body systems. We have accelerated

I Other than a neuroanatomical bias, I am politically a Libertarian and religiously an agnostic and have no a priori position on capital punishment. 
at warp speed through the issue of Descartes' Error ${ }^{2}$ to a thesis published recently in arguably one of the top two scientific journals in the world that the universe itself is all at once immaterial, mental, and spiritual, ${ }^{3}$ and a place where the physics of the material universe falls disappointingly short of a soul-satisfying explanation. This concept, especially disquieting to a wet laboratory scientist, implies that no one knows what mind, material, and brain are all about anyway.

Despite these recent intellectual upheavals, there are some useful perspectives on how neuroanatomical knowledge, combined with the new fields of brain imaging, imaging genetics, psychopharmacology, and biological psychiatry, may offer significant insights into the biological bases of the range of normal behavior, and where this normality transits or leaps into psychopathology. A recent compendium of excellent papers has used the neuroscientific approach to understanding psychopathology especially as it relates to criminality and the law. ${ }^{4}$ Rather than review that available material, I will focus on the fundamental principles of neuroanatomy and selected clinical cases in psychopathology so that the non-expert may better understand what genetic, epigenetic, neurochemical, and early brain events are associated with violent psychopathology that manifests itself later in puberty and adolescence.

This article will begin with a section on the simplest description of the neurological deficit most commonly found in psychopaths, including those with violent histories. This information is intended for those who prefer a "bottom line" summary of the neuroanatomical basis of psychopathology. Following this summary will be a more detailed discussion of the range of potential interacting biological causes of neuropathologies associated with psychopathic behaviors and some thoughts on how to interpret and challenge these biological explanations.

\section{THE BOTTOM LINE}

The simplest explanation, and most common finding, for the biological basis of psychopathology especially associated with impulsive and violent behavior is that the individual has incurred significant damage to the orbital cortex and adjacent parts of the prefrontal and anterior temporal lobe. The orbital cortex is that part of the prefrontal cortex that is positioned directly over the orbits or eye sockets. It is located at the base of the frontal lobe as illustrated in Figures 1 and 2. In addition to the core involvement of the orbital cortex, other brain areas also have been implicated in numerous neurological and imaging studies of violent, aggressive, and impulsive psychopaths. These include the frontal pole, ventromedial prefrontal cortex, ventral anterior cingulate cortex, and the

2 See antonio Damasio, Descartes' Error: Emotion, Reason and the Human Brain (1994).

See Richard Henry, The Mental Universe, 436 NATURE 29, 29 (2005).

4 See The Royal Society Theme Issue, Law and the Brain, 359 PHIL. Transactions R. Soc'Y LoNDON B No. 1451, 1657-809 (S. Zeki \& O.R. Goodenough eds., 2004). 
anteromedial temporal lobe structures such as the amygdala, temporal tip, anterior parahippocampal gyrus and hippocampal area. ${ }^{5}$

Figure 1(A).

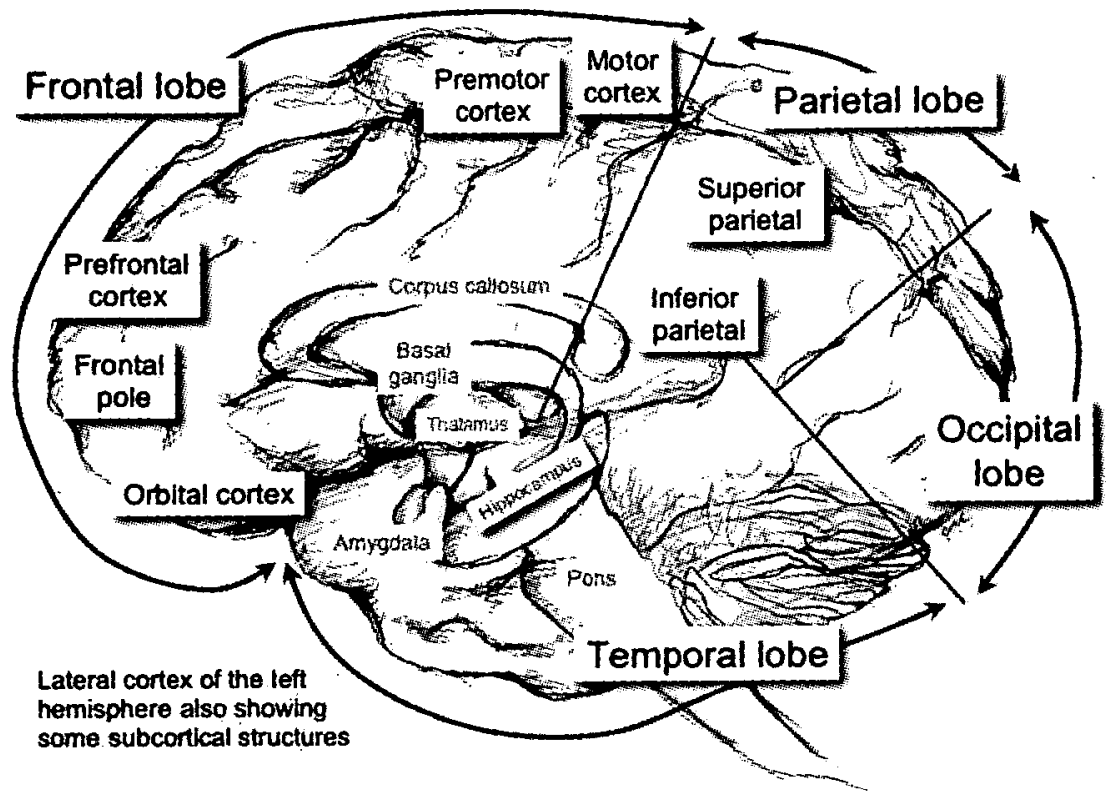

5 See Jordan Grafman et al., Frontal Lobe Injuries, Violence, and Aggression: A Report of the Vietnam Head Injury Study, 46 NEUROLogY 1231, 1231-38 (1996); Mary Best et al., Evidence for a Dysfunctional Prefrontal Circuit in Patients with an Impulsive Aggressive Disorder, 99 PROC. NAT'L ACAD. SCl. 8448, 8448-53 (2002); R.J. R. Blair, Neurocognitive Models of Aggression, the Antipersonality Disorders, and Psychopathy, 71 J. NEUROLOGY NEUROSURGERY PSYCHIATRY 727, 727-31 (2001); Adrian Raine et al., Reduced Prefrontal and Increased Subcortical Functioning Assessed Using Positron Emission Tomography in Predatory and Affective Murderers, 16 BEHAV. SCI. \& L. 319, 319-32 (1998). See generally M.C. Browser \& B.H. Price, Neuropsychiatry and Frontal Lobe Dysfunction in Violent and Criminal Behavior: A Critical Review, 71 J. NEUROLOGY, NEUROSURGERY, \& PSYChIATRY 720, 720-26 (2001). See Jeffrey M. Burns \& Russell H. Swerdlow, Right Orbitofrontal Tumor with Pedophilia Symptom and Constructional Apraxia Sign, 60 ARCHIES OF NEUROLOGY 437, 437-40 (2003); Jorge Moll et al., The Neural Correlates of Moral Sensitivity: A Functional Magnetic Resonance Imaging Investigation of Basic and Moral Emotions, $22 \mathrm{~J}$. NEURosCIENCE 2730, 2730-36 (2002); Antoine Bechara, The Role of Emotion in Decision-Making: Evidence from Neurological Patients with Orbitofrontal Damage, 55 BRAIN \& COGNITION 30, $30-40$ (2004); R. James R. Blair, Neurobiological Basis of Psychopathy, 182 BRITISH J. PsYchIATRY 5, 5-7 (2003); Henrik Soderstrom et al., Reduced Frontotemporal Perfusion in Psychopathic Personality, 114 PsYChIATRY RES. NeUROIMAGING 81, 81-94 (2002); Nathalie Camille et al., The Involvement of the Orbitofrontal Cortex in the Experience of Regret, 304 SCIENCE 1167, 1167-70 (2004); J. Homack et al., Changes in Emotion After Circumscribed Surgical Lesions of the Orbitofrontal and Cingulate Cortices, 126 BRAIN 1691, 1691-712 (2003). 
Figure 1(A) is an illustration of the lateral cortical surface of the hemisphere and some underlying subcortical structures such as the corpus callosum, the white matter bundle connecting the left and right hemispheres, the basal ganglia, thalamus, hippocampus (a memory-related cortical structure in the temporal lobe), amygdala, and pons. The cortical part of the hemisphere is subdivided into four lobes, the temporal, occipital, parietal, and frontal lobes. The frontal lobe is subdivided into the motor cortex for executing movements, the premotor cortex for the planning of movements, and the prefrontal cortex for planning, will, executive/short-term memory, personality, and a myriad of other complex adaptive behaviors. The prefrontal cortex is subdivided into around a dozen sub-regions.

Figure 1(B).

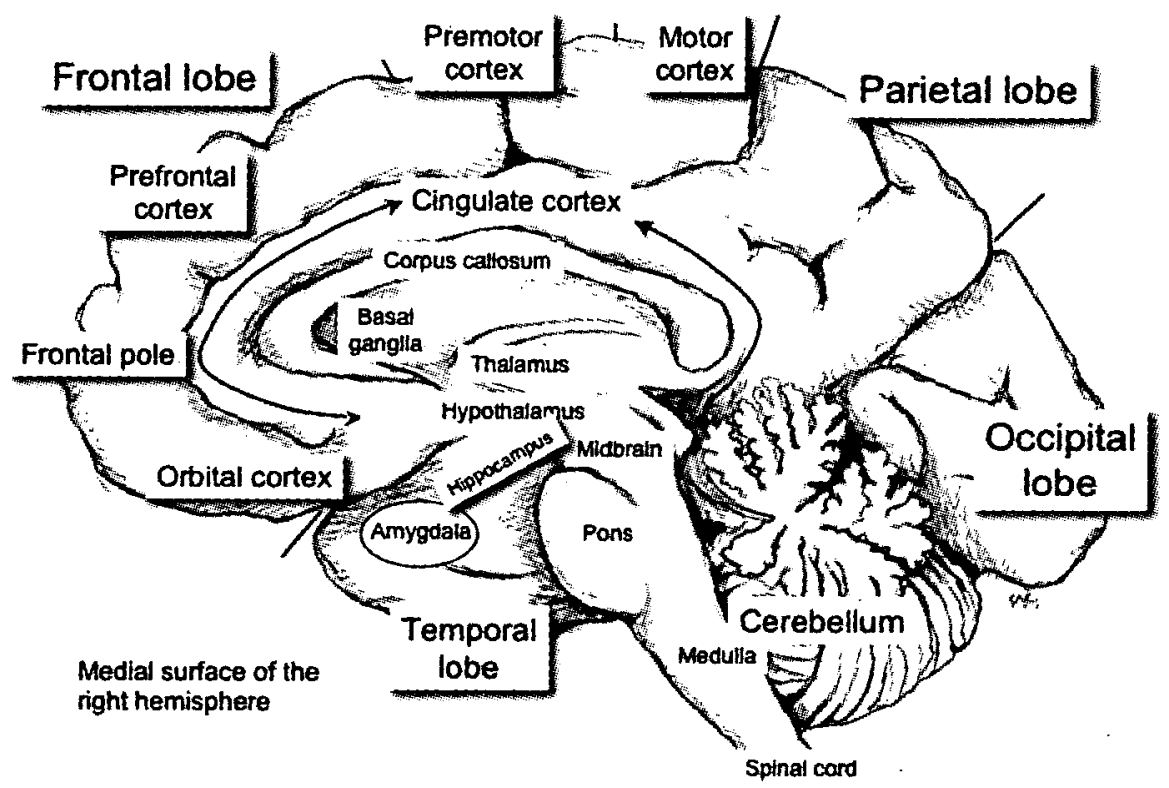

The orbital cortex, among other things, is involved in social interactions, inhibition of impulsive behavior, ethics, morality, reward and punishment, regret, and projection of future outcomes based on implied expectations of planned nearterm actions. See Figure 1(B). Adjacent prefrontal areas such as the frontal pole are also cited in psychopathology literature, as are the ventromedial cortex and ventral anterior cingulate, which are best appreciated on a medial view of the hemisphere. In addition to these prefrontal areas, the anteromedial temporal lobe, including the temporal tip, amygdala, and parahippocampal gyrus, are also implicated in the etiology of violent psychopathology. See Figure 2. 
Figures 2(A) \& (B).

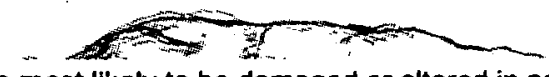

Brain areas most likely to be damaged or altered in psychopaths
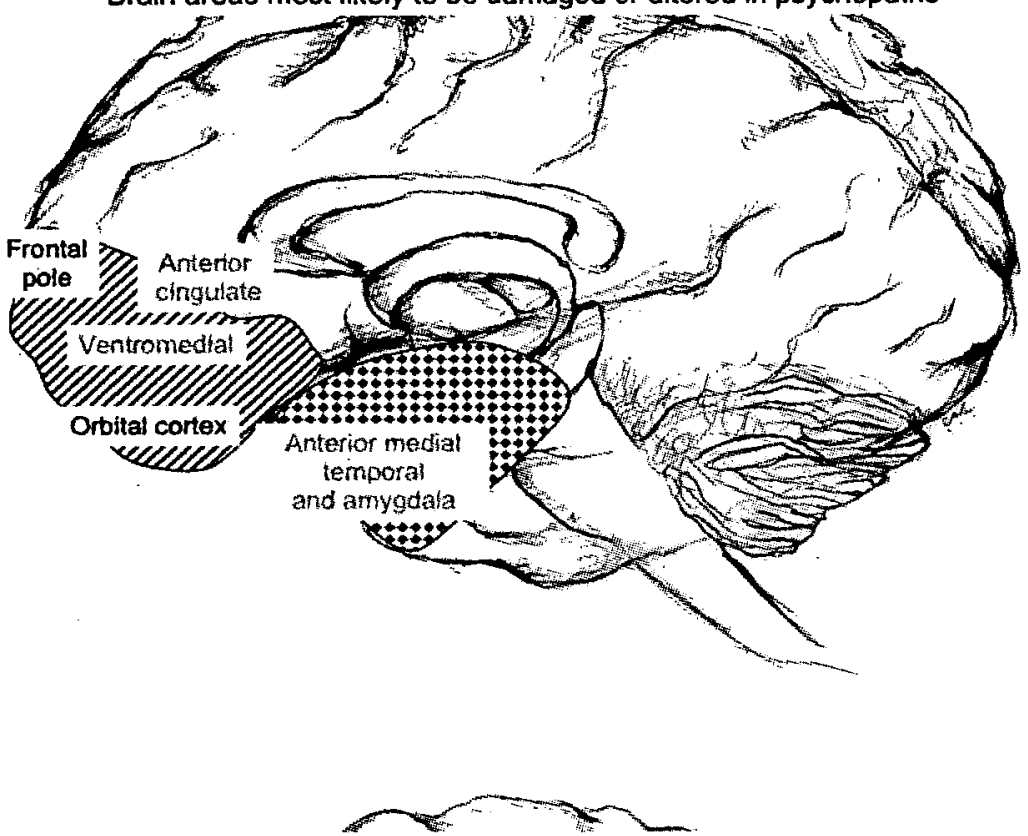

Brain areas most likely to be damaged or altered in psychopaths

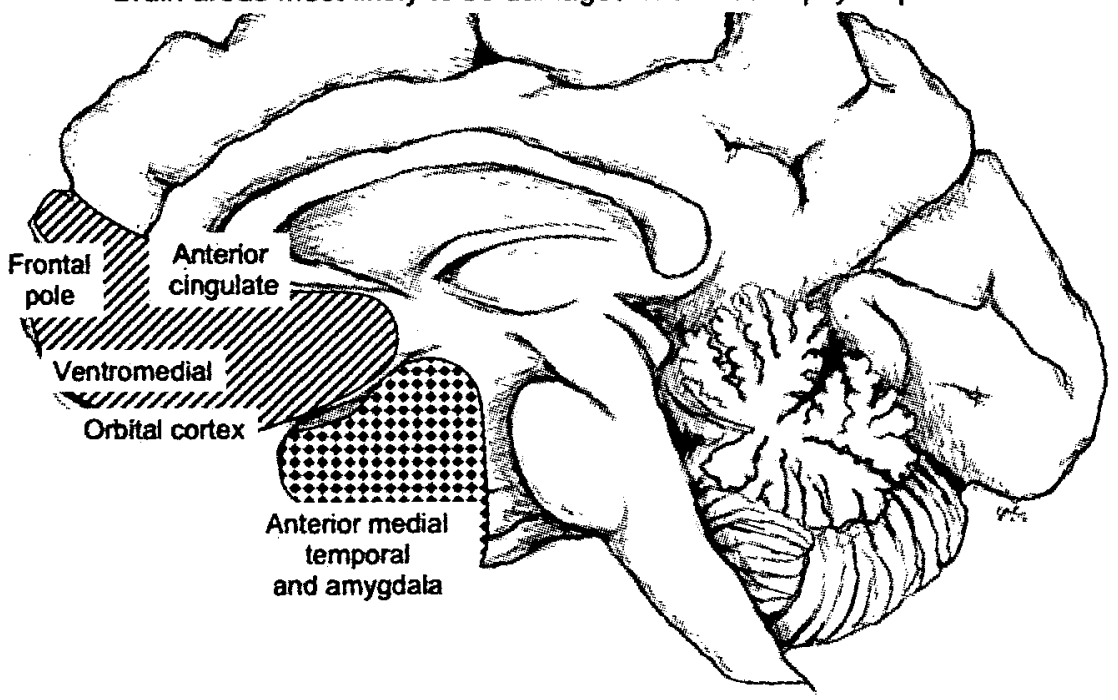


Lateral (Figure 2(A)) and medial (Figure 2(B)) views of the brain show the areas most likely implied in the etiology of at least some, and perhaps most, forms of psychopathology. These areas of the prefrontal cortex include principally the orbital cortex, and to some degree, the frontal pole, ventromedial cortex, ventral anterior cingulate cortex, and the amygdala, temporal pole, and parahippocampal gyrus in the anteromedial temporal lobe. As pointed out in Section III, it is not simply that these areas are grossly damaged, but that the circuitry connecting these areas with each other and with several key regions, are either interrupted by mechanical or toxic damage, or dysregulated by several endogenous factors. These factors may include abnormal neurotransmitter systems, stress hormones, and other gene products that may be nascent prior to birth and childhood, but only manifest themselves with later neural and endocrine development and inevitable environmental challenge.

The explanation of why these areas, especially the orbital cortex, are particularly involved in psychopathology is rather complex even for a seasoned neuroscientist, but some basic points can be made. First, the orbital cortex is involved in the regulation of many social functions, which include what we commonly call ethics and morality. This same prefrontal region also generally inhibits behavior. Thus, the orbital cortex and adjacent structures, especially on the ventromedial surface of the hemisphere, also are implicated in the control of aggression and violence, as well as appetitive behaviors like eating, drinking, sex, and other acts prone to addiction and abuse. Therefore, if the orbital cortex is broadly damaged, especially if the damage is in both hemispheres, then the individual is much less capable of inhibiting aggression, violence and addictions. If these behaviors impact others to some arbitrarily defined degree, then the behavior is variably termed immoral, unethical, antisocial, and/or criminal.

The extent of damage, the degree of bilaterality of the damage, the age of the damage, and the patency of other brain regions will impact the severity and permanence of any ensuing psychopathic behaviors. For example, smaller lesions in one hemisphere of the brain in younger children are thought to have a relatively good prognosis. However, bilateral lesions in the brain of toddlers can have longterm devastating behavioral effects that may not become obvious until the child reaches adolescence or young adulthood.

In general, there are two basic types of retrospectively determined sociopathy/conduct disorders leading to persistent criminal behavior in young adulthood. These are childhood-onset and adolescent-onset variants. The presence of a delayed trajectory also may differ by gender, with boys displaying childhoodonset and adolescent-onset trajectories ${ }^{6}$ and girls displaying both childhood-onset and delayed-onset pathways. While many of these psychopathology-prone

6 See Benjamin B. Lahey et al., Four-Year Longitudinal Study of Conduct Disorder in Boys: Patterns and Predictors of Persistence, 104 J. ABNORMAL PSYCHOL. 83, 83-93 (1995).

7 See Persephanie Silverthorn \& Paul J. Frick, Developmental Pathways to Antisocial Behavior: The Delayed-Onset Pathway in Girls, 11 DEV. \& PSYChOPATHOLOGY 101, 101-26 (1999). 
children have not undergone exhaustive imaging, genetic, and long-term clinical longitudinal follow-up, a low-range variability and blunting of the cortisol response has provided a generally reliable marker, especially in boys. ${ }^{8}$

\section{THE PRoblem BeCOMES PROBLEMATIC}

There are several serious problems with the "damaged brain area" approach to understanding the basis of normal and psychopathic behavior. Among them is the incorrect assumption that one brain structure is solely responsible for the elaboration of a complex adaptive behavior. Thus, although neurologists and psychophysicists can ascribe specific functions resident in the primary sensory cortex, or motor cortex, as the function becomes more complex, the less it is completely controlled by one brain area. In addition, there are confounding problems when a brain area is damaged. First, if there is a vascular lesion (e.g., a stroke), then several brain areas are compromised, limiting the ability to assign causality of symptoms to one area. Second, when even just one brain area is thought to be damaged, other distant areas also are affected, since fiber bundles passing through or near to the brain area of interest and projecting to distant sites are damaged. This "false localization" problem is caused by destroying "fibers of passage." It is most noticeable with some lesions of the frontal lobe, especially the posterior orbital and ventromedial and cingulate cortices.

8 See Keith McBurnett et al., Low Salivary Cortisol and Persistent Aggression in Boys Referred for Disruptive Behavior, 57 ARCHIVES GEN. PSYCHIATRY 38, 38-42 (2000). 
Figure 3. False localization of lesions and behavior and the "en passage" fiber bundle problem.

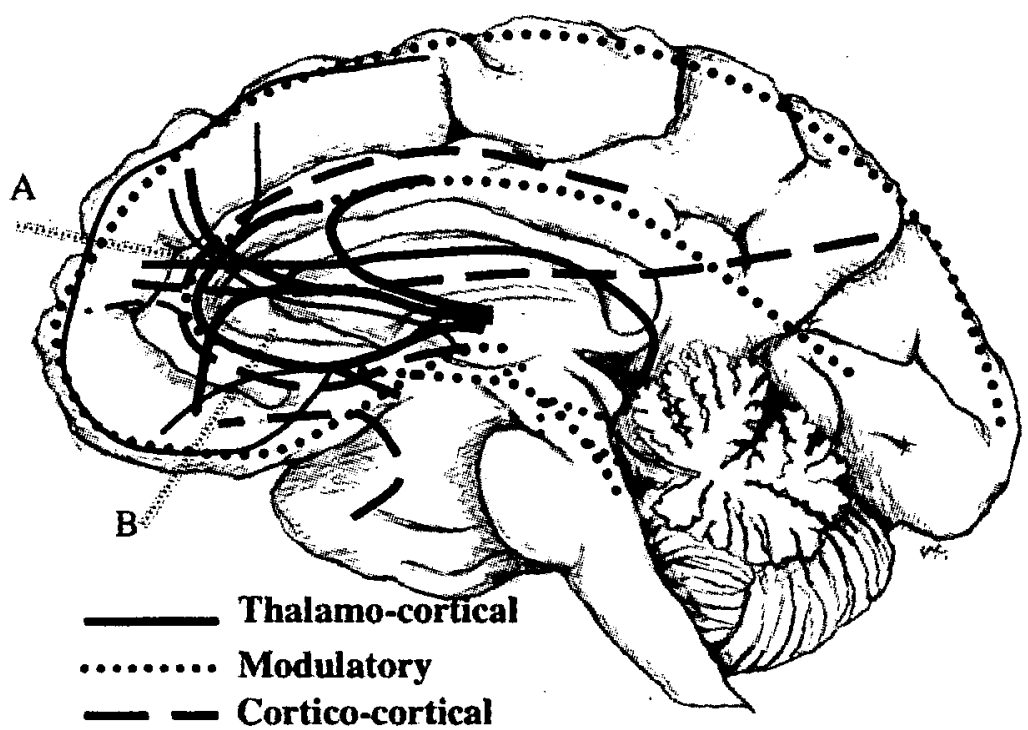

Figure 3 is an illustration of how these tracts might variably be severed given two different frontal lobotomies or sites of brain damage at frontal lobe sites $\mathrm{A}$ and B. Note that even though the frontal lobotomies are close to each other, the fiber tracts cut in each case are very different and would be expected to produce very different symptoms and pathologies. The lesion at B cutting through not only the orbital, ventromedial, and anterior cingulate cortices, but also the more widely projecting fiber bundles would be more likely to lead to psychopathic behavior. In addition to the false localization problem, lesions in the same brain regions at different ages will produce different symptoms. Not only do each of the brain areas mature at different rates, with the dorsal (upper) prefrontal cortex and frontal pole, and portions of the language cortices maturing the latest in development, even into the early and mid twenties, but the fiber bundles themselves mature (myelinate) at different rates, as illustrated in Figure 4. 
Figure 4. Progression of cortical areas and myelination of the fiber bundles connecting these areas with other brain regions.

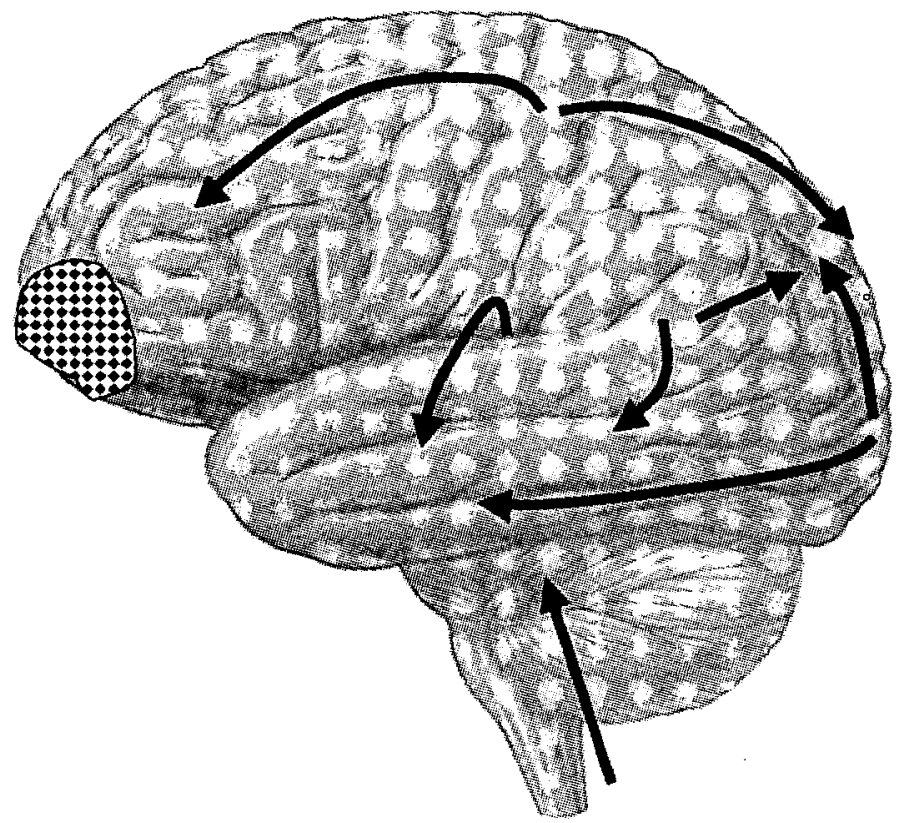

Note that the first areas to mature are the sensory and motor cortices, followed by nearby association cortices and lastly by prefrontal and language cortices (arrow tips are last to develop). Also last to develop is the 'final' (development is never really over in the brain, however') pattern of innervation of the prefrontal cortex by dopamine axons, which, like norepinephrine and serotonin axons, are unmyelinated. It is interesting that late-developing neurological disorders like schizophrenia and depression are closely linked to the proper functioning of the monoamines, dopamine, and serotonin, in the prefrontal and temporal cortices. These monoamine axons, like their target areas, do not fully develop until humans are eighteen to twenty five years old, when these and other thought and affective disorders are most likely to present as first episodes. The delayed onset of these and other thought and behavioral disorders was formerly believed to prove that these diseases were environmentally based. Now it is becoming clearer that these disorders have a profound genetic or epigenetic basis, despite their protracted onset very late in adolescence and early adulthood.

9 W.R. Shankle et al., Approximate Doubling of Numbers of Neurons in the Postnatal Human Cerebral Cortex and in 35 Specific Cytoarchitectural Areas from Birth to 72 Months, 2 PEDIATRIC \& DEV. PATHOLOGY 224, 244-59 (1999). 
Another problem with the "bottom line" approach to asking what single brain area is damaged in psychopathic murderers can be seen when trying to analyze which brain areas are involved in neurological disorders involving judgment, emotion, thought, and social interactions. Take, for example, the case of schizophrenia. There are now thought to be many kinds of "schizophrenias," much like there are fundamentally different kinds of psychopaths. Those neuroscientists more comfortable with dealing with infinite shades of meaning rather than with neat categories (I prefer neat categories for their clarity), contend that schizophrenia, like psychopathology, is a kind of spectrum disorder where each individual patient is like no other, but rather exhibits unique, individualized signs and symptoms to different degrees. While true, this approach may be less welcome in a court of law than in an experimental psychiatric laboratory.

Nonetheless, it may become more likely in the next decade for each person, including those with schizophrenia and perhaps violent psychopathology, to have a unique and bewildering combination of genetic variants and neural capacity which defy precise categorization. But that is exactly what is happening in the new field of imaging genetics, ${ }^{10}$ which combines genetic data from individuals and populations, structural and functional brain imaging data, clinical and psychometric profiles, and sophisticated statistical and data mining techniques to determine so-called "endophenotypes." These are intermediate phenotypes, somewhere between what a person looks and acts like, and their genotype. The endophenotype is now being used to study and predict who will most likely develop schizophrenia (well before they've even had their first episode), which individuals are most likely to have a first episode but never develop chronic schizophrenic relapses, and so forth. It is likely that the imaging-genetics approach and the endophenotype concept will be first applied in court to determine the culpability and treatment of violent psychopaths.

When neuroscientists consider a complex disorder (or set of disorders) such as schizophrenia, even the most modest approaches, such as the one illustrated in Figure 5, can get out of hand very quickly. This figure, although perhaps as simple to navigate through as a Jackson Pollock painting, is really an embarrassingly gross simplification of the functional neuroanatomy of schizophrenia. But despite the blinding opacity of Figure 5, one can still come to the reasonable and tenable conclusion that there are many roads to schizophrenia, because there are many direct ways to impact brain structure. In this analysis, for example, it appears that the common mode target structure for the elaboration of schizophrenia is quite

10 See Joseph H. Callicott et al., Variation in DISCl Affects Hippocampal Structure and Function and Increases Risk for Schizophrenia, 102 PROC. NAT'L ACAD. SCI. 8627, 8627-32 (2005); Andreas Meyer-Lindenberg et al., Midbrain Dopamine and Prefrontal Function in Humans: Interaction and Modulation by COMT Genotype, 8 NATURE NeUROSCIENCE 594, 594-96 (2005); Lukas Pezawas et al., 5-HTTLPR Polymorphism Impacts Human Cingulate-Amygdala Interactions: A Genetic Susceptibility Mechanism for Depression, 8 NATURE NEUROSCIENCE 828, 828-34 (2005); J.A. Turner et al., Imaging Phenotypes and Genotypes in Schizophrenia, 4 NeUROSCIENCE 21, 21 (2005). 
focused: the pyramidal output neuron, primarily in the dorsal prefrontal cortex. This dysregulation may occur through imbalance effected through a multitude of converging pathways. Extrapolated to the etiology of violent psychopathic behavior, there may be multiple pathways that may all seem different, but many may lead to the orbital and adjacent ventral and medial prefrontal and anterior temporal cortices.

Figure 5. Summary circuit diagram of the connections, revealed by replicated studies, of the connections implied in the etiology of schizophrenia."

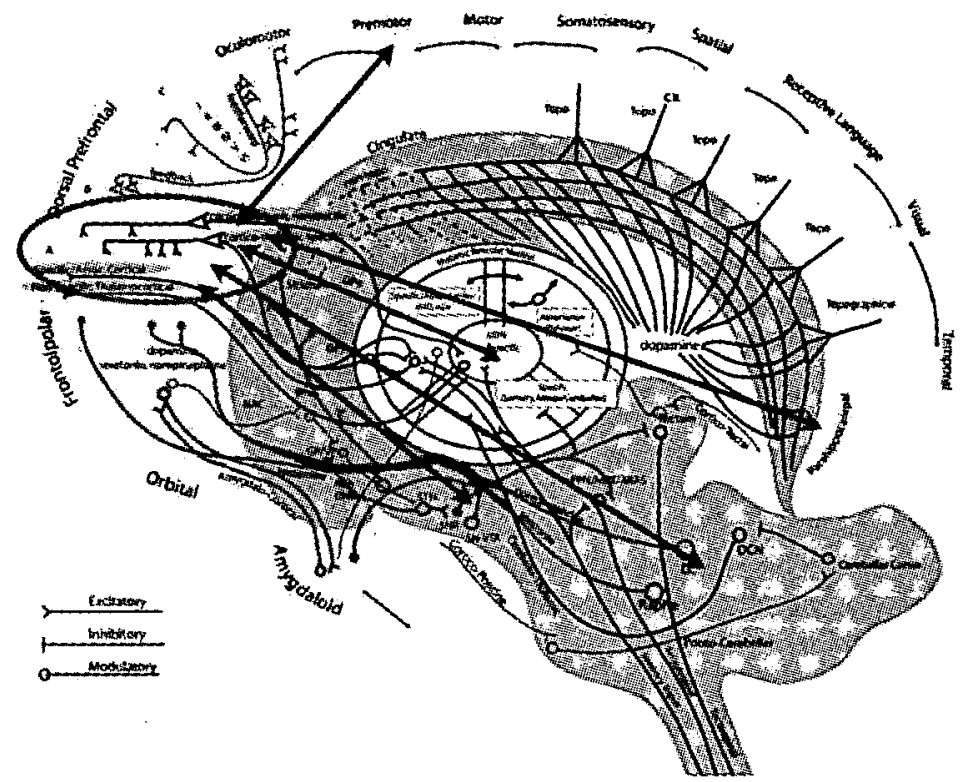

Convergence onto the final common pathway on the Prefrontal Cortex pyramidal neuron

The type of analysis depicted in Figure 5 is called a "neuroanatomical circuit analysis" or "connectional analysis." It is often combined with knowledge of neurochemistry (neurotransmitter and hormonal communication systems between cells), psychopharmacology (drug action on the circuits and behavior), and genetic analyses to generate a more complete "functional neuroanatomical analysis."

Although there are numerous caveats to defining focal, organic neural causes of psychopathology, the simple region-of-interest (e.g., orbital cortex damage) neuroanatomical analysis from clinical lesion studies in violent, overly disinhibited

11 See James H. Fallon et al., The Neuroanatomy of Schizophrenia: Circuitry and Neurotransmitter Systems, 3 CLINICAL NEUROSCIENCE RES. 77, 77-107 (2003). 
adolescents and adults is a still a good first order starting place. But one may better interpret lesions or dysfunction of these regions by noting that the age and extent and bilaterality of the brain lesion or dysfunction (e.g., by faulty neurotransmitter systems, white matter problems, in utero developmental problems) are critically important. The systems context of the brain injury also must be considered, and considered at multiple levels of brain organization from genes to broad neural circuitry.

\section{FROM GENES TO NEUROTRANSMITTERS TO NEURONS TO SYNAPSES TO CIRCUITS TO BEHAVIOR}

Although there is no clear consensus among neuroscientists on how behavior is effected through activity in the nervous system, a series of general statements can be made. Briefly (see Figure 6 for more explanation), behaviors "emerge" through interactions at many levels of resolution in the nervous system, and between the nervous system and the other systems in the body such as the endocrine and muscular systems. The interactions are mediated by connections between neurons called synapses, where one or more of 200 neurotransmitters, and related 1000 plus molecules, provide the neurochemical link in the circuitry. If the anatomical links in the circuit change, or the functioning of the neurotransmitters changes, the function of the circuit and the behaviors associated with it will change. Thus, by saying "dopamine controls motivated behavior," one is implying that dopamine is primary to the function. But this simple statement also needs to be interpreted in the sense that many other molecules such as protein receptors and second and third messenger systems are involved, and that these interact with other neurotransmitters, cell clusters, and circuitry systems to impact the behavior. When one says "dopamine dysfunction is at the root cause of schizophrenia," one understands this to really mean that multiple dopamine receptors and their downstream and upstream cascades, which are controlled by many genes (which themselves interact), impact multiple synapses and other neurotransmitter systems in at least several brain areas to influence some symptoms of schizophrenia. ${ }^{12}$

The interactions in nervous tissue that create and modulate behavior are organized in distributed neural networks that are also called "circuits" or "systems." These circuits, which are connected to other circuit modules, begin to be laid down early in brain development-for example, during the second and third trimesters of fetal life. These circuits are generally inherited from five million years of hominid evolution so that we are probably born with these pre-

12 These fine points of understanding quickly expand by two orders of magnitude more mind numbing in the primary neuroscience literature, so it is very difficult for any one scientist to get his arms around the problem with a cogent explanation. I would suspect that all the levels of complexity could become much too much for any intelligent juror or attorney or judge to handle as well, and even under the most favorable of ethical intentions, become useless in a court of law unless the concepts are greatly simplified, without becoming completely wrong at the same time. 
wired patterns in our brain circuitry. These patterns manifest themselves in many ways, like a fear of snakes, a pan-cultural appreciation of beauty, or repulsion. The circuits are laid down sequentially so that some circuits are nearly completed by the age of three while others do not fully mature until we are around twenty years of age. Other behaviors also emerge throughout perinatal and postnatal development in a sequence that is subsequently quite resistant to environmental influences.

Although there are, perhaps, many thousands of "standard" behaviors we develop as humans, we highlight several hundred "complex adaptive behaviors" such as walking upright, social smiling, and fear of strangers. These behaviors are elaborated through several interacting circuits or "loops" in the brain which ultimately control thought, emotion, muscles, glands, and autonomics. In turn, this allows us to interact with the environment, other people, and in social groups. These complex adaptive behaviors solidify during "critical periods" of development that occur in specific time periods from birth to about twenty years of age (and for some, even later in life). These maturing circuits can continue to be molded to a minor degree throughout life as we learn, create new memories, etc. Some of this remodeling involves sculpturing of the same neurons, while some remodeling may be due to the addition of new neurons into the circuits and myelination of their axons postnatally and probably for over twenty years to life. ${ }^{13}$

13 Shankle et al., supra note 9. 
Figures 6(A) \&(D).
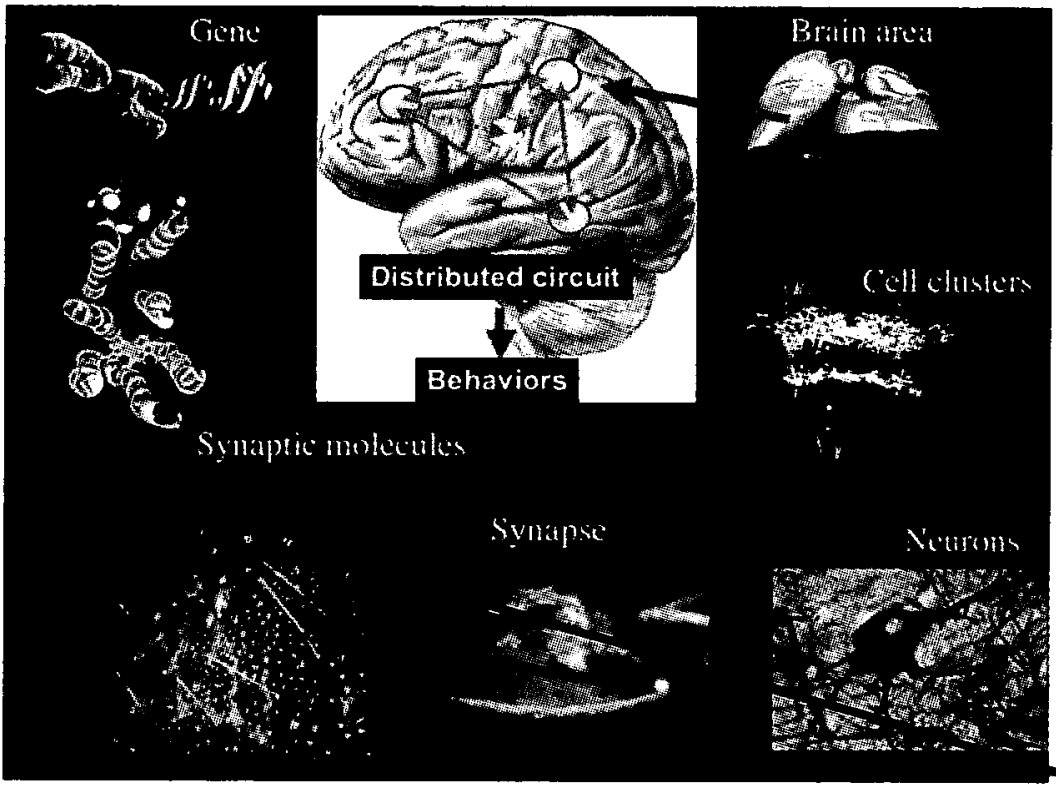

Fundamental Input, Output, Intrinsic Connections

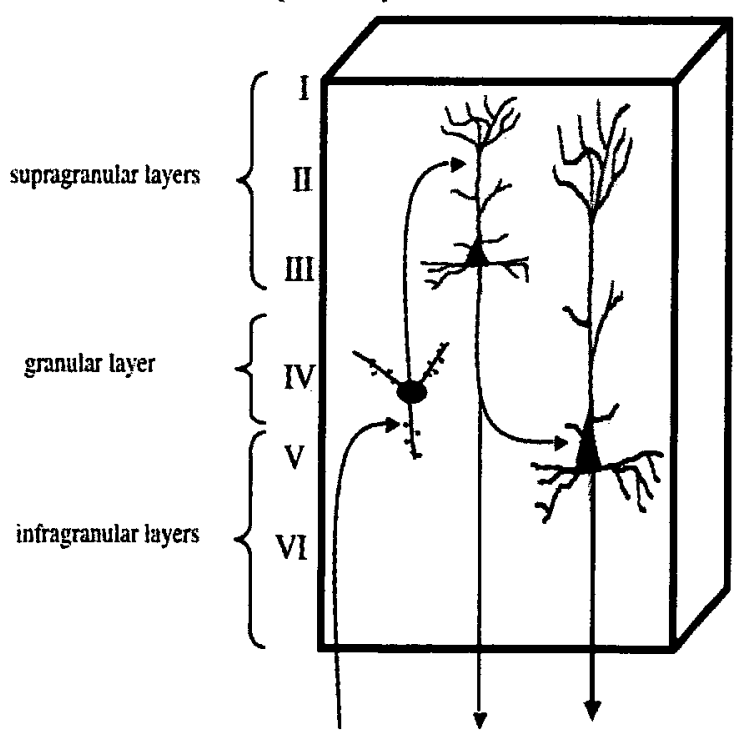


Figures 6(B) \& (C).
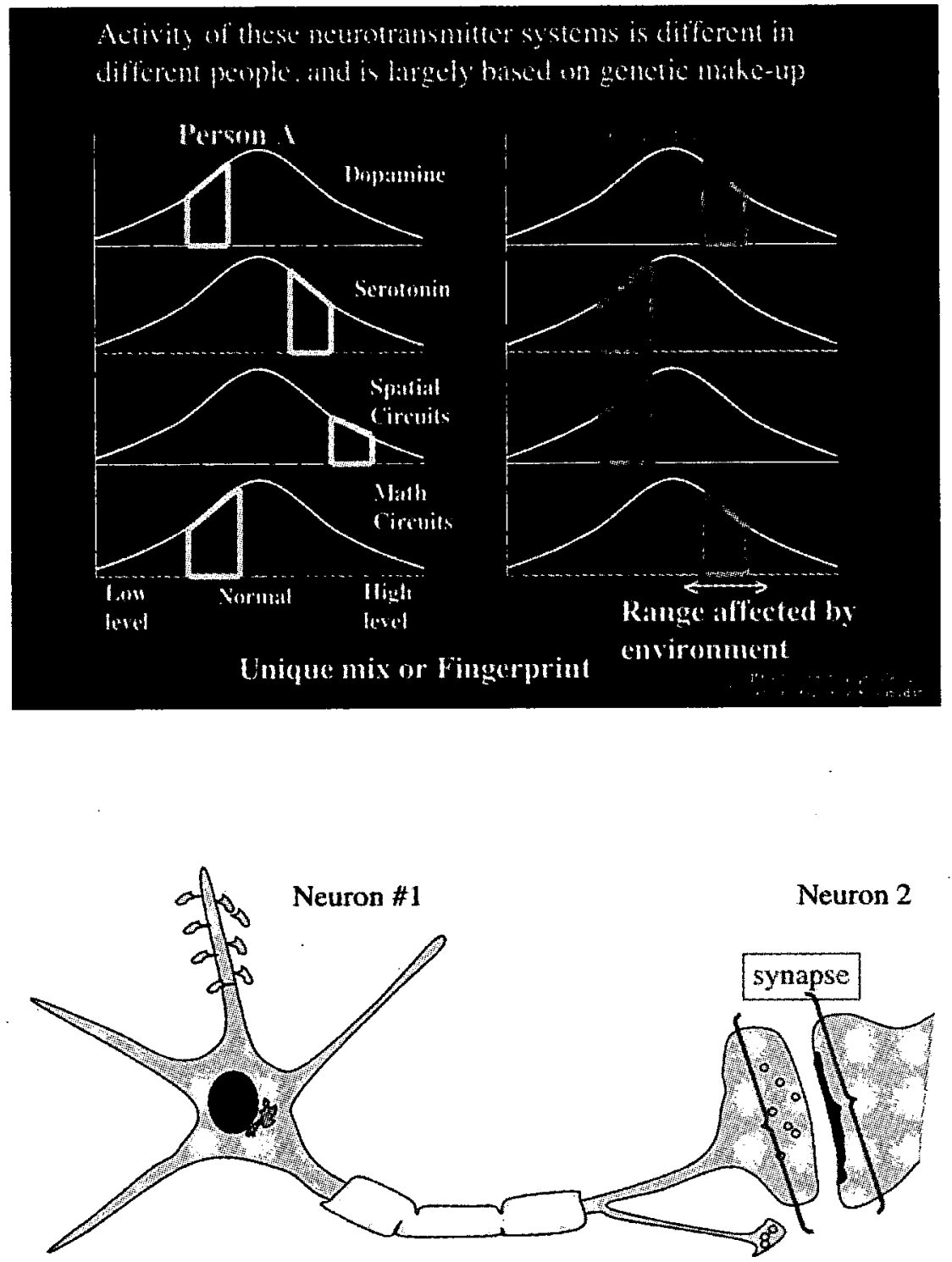

The illustration in Figure 6(A) highlights the levels of organization in the brain from the $\sim 20,000$ genes to the $\sim 200$ key neurotransmitters and thousands of 
neurotransmission-related molecules coded from these genes, to the $\sim 1,000$ to 500,000 synapses per neuron, to the forty billion neurons, arranged in cell clusters, to large brain nuclei and cortical regions, to modules of looping distributed feedback and feedforward circuits which interact in some way to effect behavior. Given an estimated 500 trillion synapses in cortex alone, there are perhaps ten billion bits of info per neuron and a virtual infinity of possible interactions among neurons in a single brain.

Figure $6(B)$ is a simplistic diagram of normally distributed ranges of hypothetically genetically controlled neurotransmitter systems and abilities in two different people, illustrating that even if all behavior is basically controlled by genes, and very little is controlled by environment, then the enormous mix and range and variety of expression patterns in individuals would still make each person in the world unique, and each would have a unique "fingerprint" of their genotype. Thus, the great diversity and range of behaviors present in the population need not be an indication of differing environmental influences.

Figure $6(C)$ depicts two or more neurons participating in forming a synapse.

Figure 6(D) illustrates groups or clusters of neurons with similar overall functions, interacting with others before sending outputs to larger modules and circuits.

\section{NEURAL CIRCUITRY AND THE IMPORTANCE OF THE ORBITAL CORTEX ENVIRONS IN PSYCHOPATHOLOGY}

The bulk of the literature and clinical findings support the notion that dysfunction of the orbital cortex bilaterally is strongly associated with impulsive and aggressive behavior and with violent psychopathology. As discussed previously, even though the orbital cortex is the most common area found to be compromised, in many cases the adjacent ventral cortices also are involved to one degree or another. These include the ventromedial prefrontal cortex, anterior cingulate cortex, frontal pole, and anteromedial temporal lobe, especially the amygdala. Examples of SPECT scans of two convicted psychopathic murderers are shown in Figure 7. 
Figure 7. SPECT Scans.

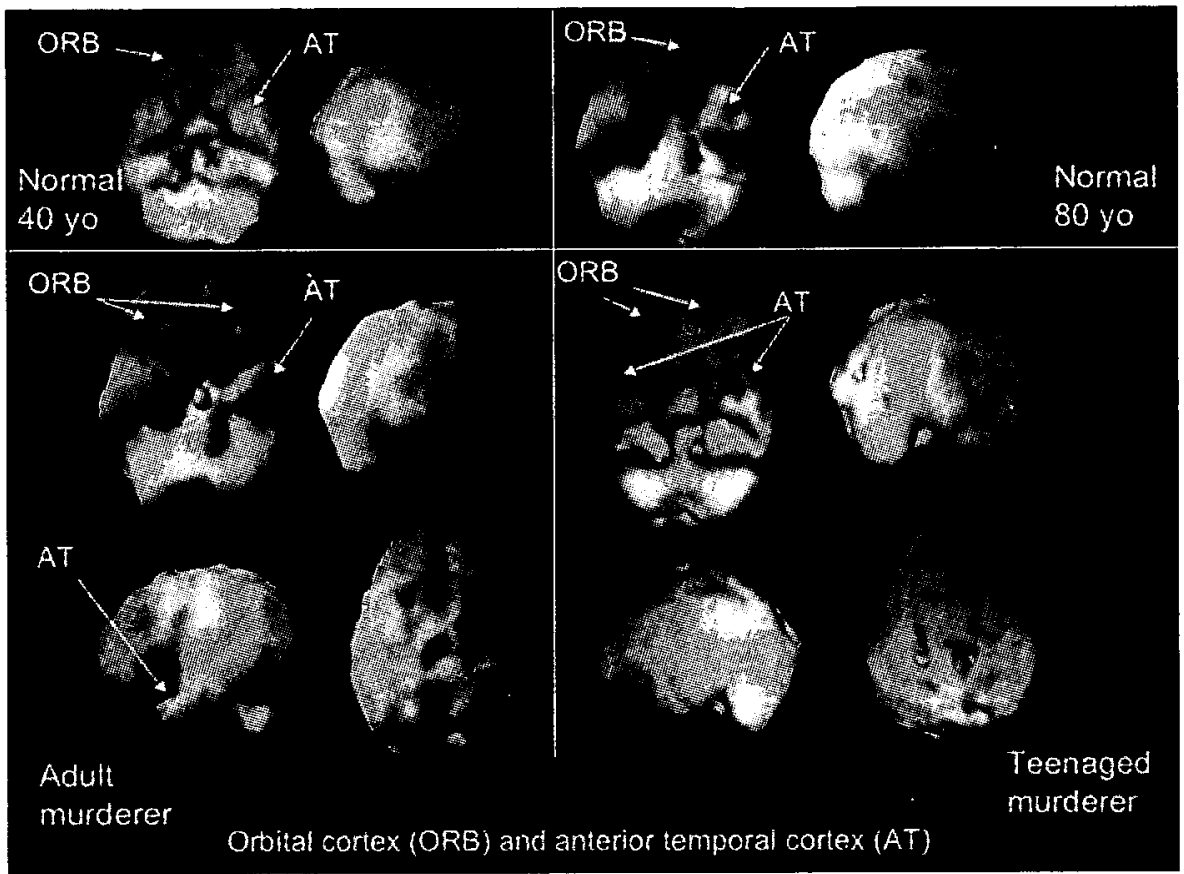

Figure 7 illustrates SPECT scans ${ }^{14}$ (a functional imaging technique similar to PET scans but measuring blood flow instead of direct metabolic activity of the brain) of two subjects: a normal forty year old (top left) and an eighty year old (top right). Note that the brain surfaces are smooth, including over the orbital cortex (ORB) and anterior temporal lobe (AT). In a young psychopathic murderer (bottom right) and adult psychopathic murderer (bottom left) the orbital cortex and anterior temporal lobes show pitted surfaces, indicating reduction or loss of function. In the teenaged murderer, there is also pitting in the posterior parietal cortex.

As discussed previously, the orbital cortex and anterior temporal lobe involvement in psychopathology should also be interpreted in light of the larger circuitry of these areas. The orbital cortex and amygdala are known to be involved in the so-called "ATO Circuit" or amygdalo-thalamic-orbital circuit as shown in Figure 8. The ATO, and its extension the Prefrontal System, is further integrated with other key brain circuits that control (and contrast) cortical systems involved in "cold cognition" (cognition having to do with the "cold facts" and not impacted to any great degree by emotion, but also aspects of self as ego) and "hot cognition" (cognition having to do more with emotion, emotional memory, impulsiveness,

14 Courtesy, Dr. D. Amen, Amen Clinics. 
passion, and social intercourse). Although there are several ways to divide these systems in the cerebral hemispheres (e.g., cold cognition vs. hot cognition; thinking vs. feeling; emotional vs. non-emotional; etc.), dichotomous contrasts are particularly useful for heuristic, experimental, and clinical reasons, if not legal considerations. For the sake of discussion here, we can consider several dichotomies, as illustrated by Figure 9 .

Figure 8.
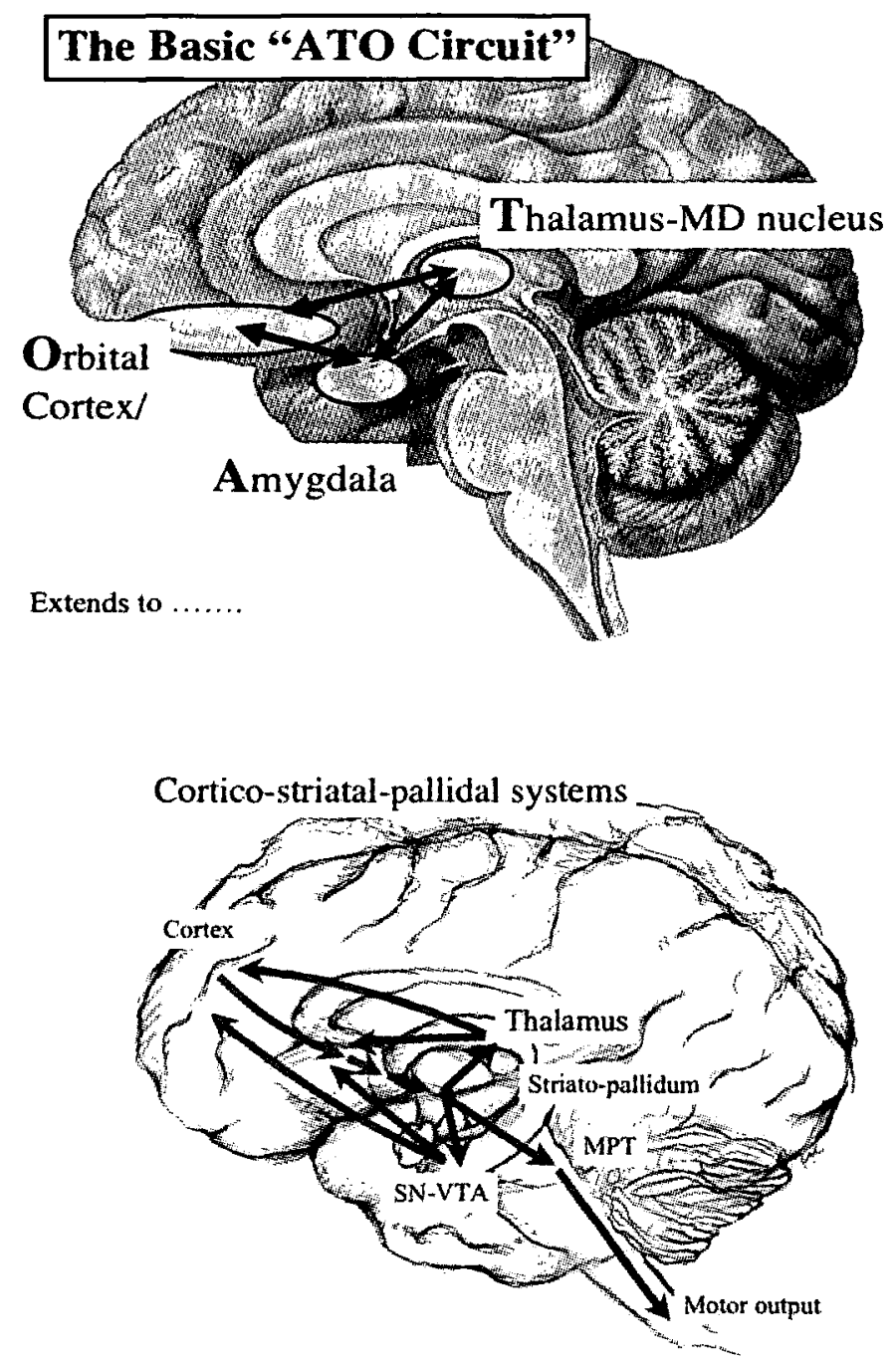
Figure 8 is the ATO or amygdalo-thalamo-orbital circuit, a "classic" circuit known for the past thirty years and which is known to participate in a wide variety of complex adaptive motivated behaviors, including addiction, emotional memory, and a wide variety of others. This basic circuit has been expanded over the past twenty years to include other tightly connected structures, including the anterior cingulate cortex, ventromedial prefrontal cortex, striato-pallidal complex, and monoamine inputs, especially dopamine and serotonin. This ventral "Prefrontal System" is involved in both cortico-cortical loops, especially with other limbic (e.g. emotional, hormonal, visceral brain) areas, as well as loop and output structures in the largest cortico-subcortical system in the brain, the cortico-striatopallidal (CSP) systems which both update cortical modules and provide outputs to motor and endocrine and autonomic nervous system structures which elaborate actual behavior (at right). The progressive open loops in these CSP also provide a means for the cognitive, thinking brain to communicate with the emotional brain.

Figures 9(A)-(D).
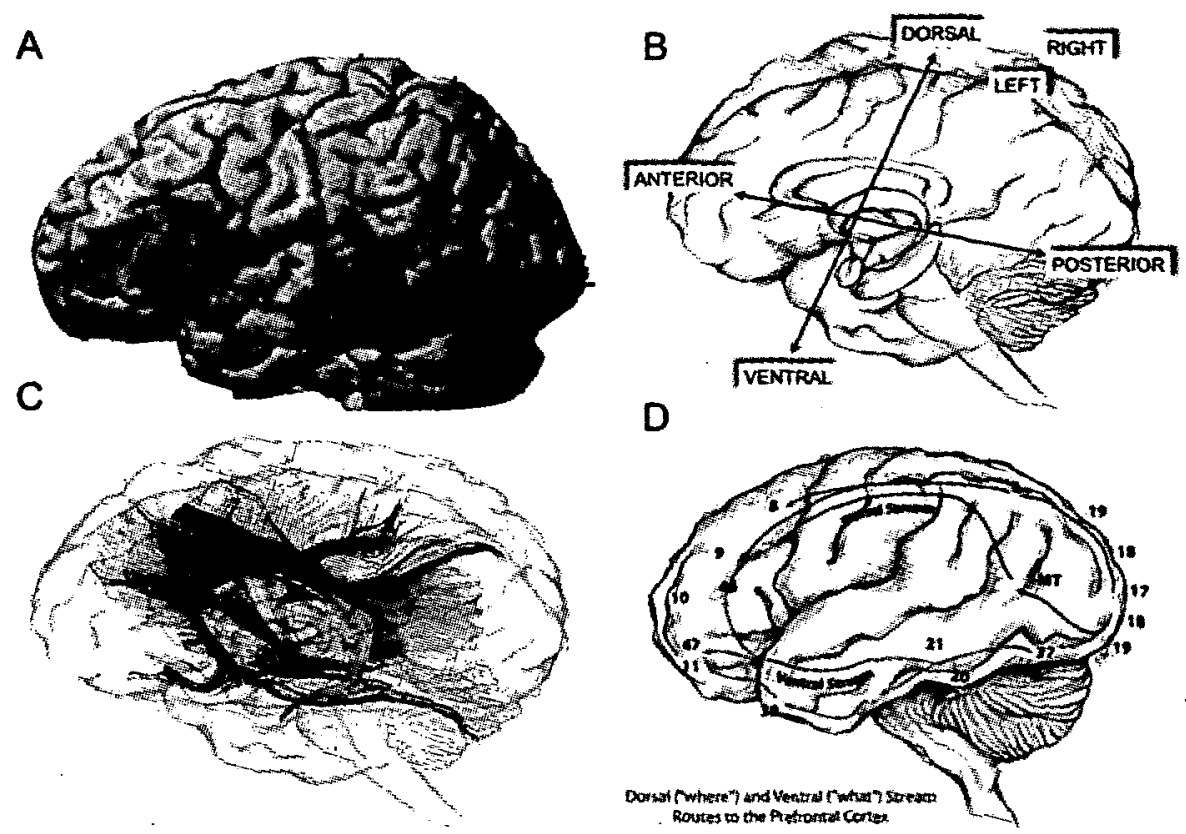

Figure 9 illustrates several points. First, in numerous neurological disorders there appear to be dichotomous or contrasting functional patterns. See Figure 9(A). For example, in patients with predominantly negative symptom schizophrenia (flat affect, poverty of speech or expression of emotion, withdrawal), the activation pattern (as seen in the PET scan) during attention is significantly lower than those patients with positive symptoms (hallucinations, 
delusions, believing one is a deity) in the lower or ventral part of the hemisphere (blue color in the PET scan). On the other hand, some upper or dorsal areas of the hemisphere have higher activation and metabolism in the negative symptom schizophrenics (red color in the PET scan). This dorsal-ventral dichotomy in brain metabolism can also be seen in normal subjects under different behaviors. This dichotomy, which is now called the "Dorsal Stream" vs. "Ventral Stream," is illustrated in Figure 9(D). The dorsal visual stream (dark arrows in D) processes visual information having to do with "where" things are in one's visual world, whereas the ventral visual stream processes "what" things are in one's visual field. So if you look up at the bench in a courtroom and see a person dressed in a funny robe, your ventral stream will tell you that it is the judge, and when the judge storms out of the courtroom, your dorsal stream tells you the judge is moving from the bench to another room at a certain direction and speed.

There is also a cognitive (dorsal) and limbic (ventral) prefrontal stream (located in the anterior half of the brain-see the anterior-posterior dichotomy in Figure 9(B)) relating the prefrontal systems. The dorsal stream processes cognitive (cold cognitive) data like "the judge is going to make an interim ruling" and the ventral prefrontal stream processes the thought "uh, oh, the judge is mad as hell at me and I feel lousy about the ruling he is about to make." In addition to the two visual streams and two prefrontal streams, the left and right hemispheres also process different aspects of the brain's interpretation of what it means when the judge storms out of the courtroom and into her chamber. When the judge leaves, you may turn to your associate and whisper "we're about to get skewered." The grammar, syntax, and simple meaning of that statement is processed in both your and your associate's dominant hemisphere ( $97 \%$ the left hemisphere), whereas the emphasis, intonation, and emotional meaning of your statement is processed in the non-dominant hemisphere, thus appropriately provoking fear or worry or laughter.

In the normal person, the dichotomous parts of the brain are appropriately in balance, but in someone who is clinically depressed, schizophrenic, or aggressive or violent, the appropriate balance is not present between all these functions. As a result, the same stimulus to a normal person and a clinically imbalanced individual produces a completely inappropriate response, especially in a social sense. To that abnormal person, the brain can be seen as processing and in the best way it can, considering the way the stimulus is interpreted by the abnormal brain, the ongoing emotional background state, the emotional and aggressive reactivity of the different areas of the brain, and so forth. In this way, a neuroscientist might see the brain's processing and resultant behavior as "perfect" in its own right (as a machine), even though the behavior may be both psychopathological and quite illegal.

Figure $9(C)$ is an illustration of reconstructed fiber tracts (white matter bundles) connecting the frontal lobe with other cortical areas in the dorsal and ventral streams in a living human subject using Diffusion Tensor Imaging (DTI), an experimental and potentially powerful variant of the MRI for non-invasive brain scanning. This technique, when combined with functional brain imaging (PET, 
fMRI, SPECT, EEG, MEG, and MRS) and genetic analyses, may soon expand the new field of "imaging genetics" from the research lab and clinical setting into the legal system.

A specific and relevant example of a dichotomy of function and associated expanded circuitry is illustrated in Figure 10. The orbital cortex ("Orbital PFC") and amygdala ("Amygdala") send strong projections to each other with the net result that they inhibit each other's activity and output to other structures, such as the nucleus accumbens (a ventral striatal basal ganglia structure which controls motor output, i.e., behavior). The orbital cortex and amygdala also project to the nucleus accumbens where they compete for control of the nucleus accumbens and therefore compete for the control of behavior. The amygdala stimulates appetitive behaviors such as sex, aggression, feeding, and eating; the orbital cortex inhibits these behaviors. The net balance of orbital cortex versus amygdala is a key to understanding regulation of behavior, especially those involving addiction, violence, sex, and the like. An important modulator of the activity in this triangle is dopamine (DA). An increase of DA release in the nucleus accumbens, through the convergence of amygdala and midbrain dopamine inputs (VTA), stimulates appetitive behaviors, whereas orbital cortex input inhibits DA release and therefore inhibits appetitive, impulsive, and aggressive behaviors.

Other adjacent brain areas also act as modulators of orbital and amygdala activation, and the more "cold cognition" functions of the dorsal prefrontal cortex (Dorsal PFC). These modulatory switches include the dorsal and ventral anterior cingulate cortex. This expanded circuit is a major determinant in moment-tomoment behavioral regulation of whether decisions are dominated by 1) thought and non-emotional reasoning and projections of consequences of planned actions into the future (so called "memory of the future"), 2) impulsive and aggressive behaviors stimulated by immediate, appetitive needs regulated by the amygdala, or 3) emotional and "hot cognition" as regulated by the inhibitory influences of the orbital cortex, and appearing as ethical, moral, and socially desirable behaviors. In a psychoanalytical sense, these three areas and associated functions resemble the ego, id, and superego, respectively. 
Figure 10. Illustration of the dichotomous roles of the amygdala and orbital cortex in regulating behavior.

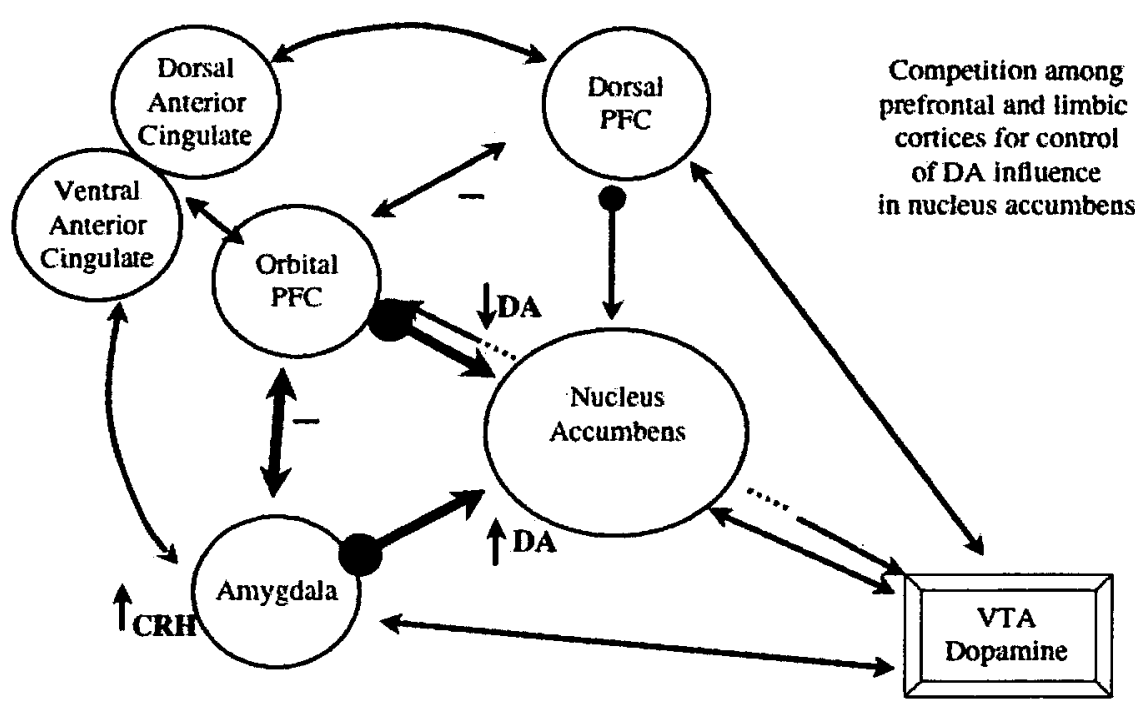

Orbital and Amygdaloid cortices inhibit each others" actions

Amygdala input increases release of DA in nuc accumbens

Orbital and dorsal PFC inputs decrease DA release in nuc accumbens.

Anterior cingulate subsectors act as mediators and indirect connectors

\section{MY TWO-YEAR-OLD GRANDDAUGHTER IS A SOCIOPATH}

Virtually everyone, under normal conditions will automatically go through genetically determined phases of development, such as those listed in Figure 11. The exact time of onset of these behaviors may vary (just as onset of puberty is different for different individuals). Everyone will also, inevitably, be barraged with a series of serious stressors as a normal part of everyday life. We may miss an important point, however, if we assume that psychotic breaks occur because of these stressors. Many neurological diseases have a primary genetic and/or prenatal development cause, but the disease may not manifest itself until decades later when the individual reaches a later developmental benchmark. For example, Alzheimer's disease may have a set of strong genetic causes but the disease does not manifest until middle age. Similarly, some types of schizophrenia may have a genetic and/or prenatal primary cause, but the disease does not manifest until late adolescence. The same is true for addiction, which has a strong genetic component. 
In all these conditions, a first episode of the disease may follow a strong stressor, such as the adolescent who has a first psychotic break late in the first semester of her first year of college. These stressors, and the inevitable availability of opportunities (e.g., drugs) at some point in an individual's life, will ultimately challenge and probably break down the individual. In the addiction field, we say that "the genes load the gun and the environment pulls the trigger." Under unusual and rare extremes, like malnourishment or nearly complete social isolation, there may not be adequate metabolic and sensory-motor/social inputs for the behaviors to be properly developed, especially during the critical periods for each behavior. But it does not really take a village for these circuits and behaviors to mature; they will develop automatically and correctly unless there are intervening catastrophic events, like brain injury or metabolic poisoning which damage specific circuits. Moreover, attempting to reverse expected causes may only preempt one minor facet of later pathophysiology. For example, enhancing the nutrition of children with poor diets may reduce the incidence of some antisocial behaviors during adolescence, but may not reduce the incidence of psychopathic homicides in this group later in adulthood. ${ }^{15}$ There are, as yet, no magic bullets-drug therapies, gene therapies, and the like-to prevent psychopathology, or to treat the psychopath effectively and humanely. There are pharmaceuticals that alter the function of monoamine and peptide neurotransmitter systems that can reduce some of the symptoms such as impulsivity and some forms of rage and aggression. There have even been reported successes in youths at risk with the daily practice of transcendental meditation. ${ }^{16}$ However, as with improved diet in starvation conditions, greatly enhanced socioeconomic status, and drug therapies, these useful environmental interventions only appear to improve co-morbid symptoms and behaviors such as stress level, cardiovascular status, impulsivity, and school infractions. These interventions do not reverse the core psychopathology associated with violence combined with a lack of remorse.

In addition, radical improvement in diet and nutrition may also ameliorate some of these behaviors, but not the chief complaint, i.e., a proclivity for seemingly unprovoked violence and murder is still untreatable.

15 See Adrian Raine et al., Effects of Environmental Enrichment at Ages 3-5 Years on Schizotypal Personality and Antisocial Behavior at Ages 17 and 23 Years, 160 AM. J. PSYCHATRY 1627, 1627-35 (2003).

16 See Mark A. Hawkins et al., Re-Enlivening and Fulfilling the Criminal Justice Rehabilitative Ideal Through the Transcendental Meditation and TM-Sidhi Programs: Primary, Secondary; and Tertiary Prevention, 17 J. SoC. Behav. \& PERSONALITY 443, 443-88 (2005); Vernon Barnes et al., Impact of Stress Reduction on Negative School Behavior in Adolescents, 1:10 HEALTH \& QUALITY OF LIFE OUTCOMES 10 (2003), available at http://www.hqlo.com/content/1/1/10. 


\section{Figure 11. Onset of adaptive behaviors in infants and children. ${ }^{17}$}

$\begin{array}{ll}\text { Onset in months } & \text { Behavior } \\ 0 & \text { Exhibits walking reflex when held upright } \\ 0 & \text { Exercises all human facial expressions } \\ 0 & \text { Distinguishes mother's breast by smell } \\ 0 & \text { Stage 1 fear to stimuli and physical support changes } \\ 0 & \text { Reflexive turning to sound } \\ 0.5 & \text { Loss of reflexive turning } \\ 0.75 & \text { Loss of walking reflex } \\ 1 & \text { Coos } \\ 1.25 & \text { Social smile } \\ 2 & \text { Laughs } \\ 3 & \text { Shows handedness preference } \\ 3.5 & \text { Discriminates mother's from father's voice } \\ 4.5 & \text { Makes raspberry sound } \\ 6 & \text { Sits alone for 30 sec or more } \\ 6 & \text { Color perception like adult } \\ 7 & \text { Loses fear 1, gets fear } 2 \text { of strangers, crowds } \\ 12 & \text { Walks alone } \\ 17 & 7-20 \text { word vocabulary } \\ 24 & \text { Adult visual acuity } \\ 30 & \text { Can climb up a tree but not down } \\ 30 & 3 \text { word sentences }\end{array}$

Some specific types of brain damage, for example to the orbital and adjacent cortices, will cause permanent malfunctioning in high order functions such as social interactions, emotional balance, and moral reasoning. The orbital cortex development is critical to socialization, and the impact of the stress steroid system is particularly important. As the orbital cortex continues to develop, social interactions become more "civilized." As a preteen, the appropriate dynamic release and response to cortisol during stress is one predictor of normal versus pathophysiological behavior. In addition, the timing of damage to the orbital cortex and associated ventral prefrontal system may be an important predictor of the specific type of psychopathology that may manifest in the preteen and adolescent periods.

If the damage occurs prior to the completion of the critical period of development for a complex adaptive behavior, the behavior may be lost forever. If the damage occurs to the circuit after the critical period of development ends, then

17 See B.H. Landing et al., The Development of Structure and Function in the Postnatal Human Cerebral Cortex from Birth to 72 Months: Changes in Thickness of Layers II and III CoRelate to the Onset of New Age-Specific Behaviors, 21 Pediatric Pathology \& Molecular MEd. $321,321-42$ (2002). 
the fundamental functions of the brain area may be present, but the brain area damaged may not interact properly with other brain areas that have not fully developed yet, thereby affecting other behaviors. One example would be damage to the orbital cortex of the frontal lobe at age two compared with age eight. Orbital cortex circuitry, and the broader system in which it participates, develop earlier (during the first two or three years of life) than other areas of prefrontal cortex such as the dorsal prefrontal cortex (which develops much later, even extending into early adulthood). The orbital cortex is involved with social interactions and the associated functions we call ethics and morality. A child with orbital cortex injury at two years of age (if the injury is extensive enough and especially if the damage is to both sides of the brain) will probably exhibit psychopathic behaviors as a preteen (childhood-onset) or teenager (adolescent-onset or delayed-onset). The type of psychopathic behavior may be very specific and profound; that is, the child/teen may commit typical psychopathic behaviors but may not, perhaps, be aware at all that what they are doing is "wrong."

A child with later damage to the orbital cortex at age eight may also exhibit similar sociopathic/psychopathic behaviors as a young teenager and may understand that what he is doing is wrong (their "morality"- sensitized orbital cortex having already developed enough early on before the brain damage occurred), but may not be able to inhibit the psychopathological impulses (the orbital cortex also inhibits impulsive behaviors throughout life through its interactions with other later developing prefrontal and temporal cortices). Someone with orbital cortex damage incurred later in life (as a later teen or adult) may still have some trouble inhibiting impulsive or aggressive behaviors, but may have enough 'help' from other areas to keep the impulses under control, as long as stressors from the external or internal environment do not become severe and protracted. Beyond considerations of outright brain damage or extreme stressors during critical developmental periods, there are the variations in behaviors that are greatly affected by variations in the expression of genes in any individual. ${ }^{18}$

The timing of neurological damage to the orbital cortex is critical, but overall, damage or genetic-molecular sequelae may result in the following changes in behavior: less strict adhering to societal rules, more daring thoughts and behaviors, experimenting with life, decreased inhibition of creativity, altered feedback to perceptual areas of the brain, loss of behavioral inhibition, altered sexuality and libido, loss of balance between immediate and delayed gratification. It may be associated with mania and/or depression, schizophrenia, obsessive-compulsive disorder, ADHD, and/or dementia. But many of these changes are not new,

18 These normal variations are called "SNPs" (single nucleotide polymorphisms). There are about 26,000 human genes (much less than the original estimates of 100,000 , now disturbingly understood to be only a slightly larger than the 20,000 found in the round worm c. elegans), but many more known SNP variants of the human genome, perhaps millions are thought to exist. Each person inherits half of their genes (as alleles) from their mother, half from their father. Of the 20,000 genes that impact the brain (and therefore behavior) some have very subtle effects and many interact with other genes (gene-gene interactions occur through a process called epitasis) to impact brain function. 
"immoral" or "evil" behaviors, but rather endogenous tendencies that are released once the inhibitory influences of the orbital cortex are removed. These released behaviors do not, however, necessarily make someone a psychopath. Precisely what combination and timing of early damage to the orbital cortex and ventral prefrontal system, and environmental triggers promote a child into a life of violent psychopathology is not yet known, but the functional neuroanatomical approach provides an important tool in the armament to predict, explain, and perhaps treat the emerging pathological killer. 
